\title{
Negotiation and Collaborative Technologies in Organisations and Supply Chains: Introduction to the Special Issue
}

\author{
Guy Camilleri $^{1}$ • Jorge E. Hernández ${ }^{2,3}$
}

Published online: 28 June 2016

(C) Springer Science+Business Media Dordrecht 2016

\section{Introduction}

Collaborative technologies refer to a set of tools for task-specific collaborations, and are associated with goal and work-oriented activities (Cheung and Vogel 2013). Collaborative initiatives such as collaborative design, collaborative planning, forecasting and open collective innovations are increasingly accepted as approaches to effectively support decision-making processes in a variety of domains, including, manufacturing, education, healthcare and software industry. Therefore, under a decision support system perspective, an effective collaboration leads to a variety of advantages, which include: better network-based partnership performance, as well as promotion of proactive decision-making processes rather than only reactive. This becomes practically valuable for organisations, particularly when they need to support their decision-making processes under heterogeneous and geographically dispersed scenarios. Given this fact, negotiations, collaborative technologies, facilitation and integration approaches are required to support the main strategic, tactic and operative decision-making processes.

$凶$ Guy Camilleri

Guy.Camilleri@irit.fr

Jorge E. Hernández

J.E.Hernandez@Liverpool.ac.uk

1 IRIT- Université de Toulouse, Université Paul Sabatier, 118 Route de Narbonne, 31062 Toulouse Cedex 9, France

2 University of Liverpool Management School, Chatham Building, Chatham Street, Liverpool L69 7ZH, UK

3 Universidad de La Frontera, Avenida Francisco Salazar, 01145 Temuco, Chile 
In addition to this, it is also true that there is a growing realisation that organisations face many challenges to cope with their decision-making processes effectively. Most of these challenges are related to the gap/mismatch in understanding what the organisation decision-making solutions really are, particularly in terms of what is offered and what the expectations of the society and industries are. This issue can be considered as a consequence of shifting from the satisfying to the "least-worst" decision-making approach. In this context, numerous studies have explored the relationships amongst decision-making processes, negotiation and facilitation with the purpose of gaining a better understanding of the key business processes in organizations (De Vreede et al. 2002; Lagroue 2008; Kwok et al. 2000). Thus, it was evidenced that, through the use of collaborative tools and negotiation approaches, organisations will get benefited by being able to support effective and assertive operational decision-making management.

In light of the above, it is important to stress the fact that the majority of the collaborative processes require consideration of the right use of skills and abilities in order to facilitate the mediation and the use of collaborative technologies within a global setting (Daniels et al. 2015). In fact, working teams will require not only to engage in a step-by-step process towards reaching a common goal, but they will also require the use of facilitators to intervene and guide the group through the collaborative process (Azadegan and Kolfschoten 2014). Under this perspective, the need of facilitation based structure becomes particularly acute for tasks that considers a longer duration and, because of their nature, are rather complex than simple, as is the case of facilitation activity based modelling (Dean et al. 1997). To accomplish this purpose, Tavella and Franco (2015) established that facilitated modelling interactions can take the form of three distinct group knowledge production patterns: generative, collaborative and assertive.

Nevertheless, even if the role and importance of facilitation, and negotiation, in group support systems are acknowledged by the scientific community, an agreement on the level of facilitation (restrictiveness) versus the facilitation content, remains elusive. The evidence as shown that it is insufficient to just create teams which will work in parallel and collaboratively to produce the modelling processes (Dean et al. 2000). Boddy et al. (2000) indicated that it is common for managers (or decision-makers) to attempt to develop collaborative alliances with other organizations. However, in the same way, they also face the challenge regarding the implementation of these alliances. This means that implementing and managing organisations' alliances might turn into a more difficult task than just establishing an effective collaboration. In fact, managerial challenges are compounded by two main aspects. The first one relates to the conflicting goals within organisations and alliances and, the second one is due to the organisational inertia caused by unnecessarily complex interactions and environmental uncertainty. Thus, technologies used for supporting the generation and management of alliances have to be adaptable to the evolving nature of these collaborative alliances. Their main goal is to support effective and assertive operations and decision management, when the organisations, as well as the alliances, are difficult to manage.

Therefore by considering the aforementioned aspects in terms of covering and supporting the current challenges for negotiation and collaborative technologies in organisations, this special issue aims to make a contribution to scientific community, researchers and practitioners, by gathering and presenting solutions to support the 
modelling and implementation of negotiation requirements, as well as collaborative technologies, in organisations based domains. This special issue provides a collection of selected papers that present novel approaches to support organisations in the following areas: communication, management, negotiation, information sharing, web based applications and supply chain management.

\section{Paper Selection}

The joint conference of the INFORMS GDN Section and the EURO Working Group on DSS was held in Toulouse in June 10th-13th 2014 and gathered 90 conference publications. In addition to the papers presented in the proceedings (Zarate et al. 2014), 12 contributions, prepared for the Negotiation and Collaborative Technologies sessions, were selected for their potential publication in in the Group Decision and Negotiation Journal. These contributions were revised and extended and underwent a two-stage and triple-blind reviewing process. The best six papers were selected for publication. These papers present novel approaches and new findings under the theme "Negotiation and collaborative technologies in organisations and supply chains". All submitted contributions were of high quality making the selection process challenging. The Guest Editors are grateful for the commitment of the authors and the reviewers during the reviewing process.

\section{Content}

The papers included in this special issue enrich the fields of negotiation and collaborative technologies. The Guest Editors greatly appreciate the interest that this special edition has generated. They also wish to thank every reviewer; without their hard work, insightful comments, and selfless support of our research community it would have not been possible to prepare this issue. The list of reviewers is presented in the acknowledgement section.

The final selected contributions are listed and briefly described as follows:

The first paper, by Dorota Górecka, Ewa Roszkowska and Tomasz Wachowicz, "The MARS approach in the verbal and holistic evaluation of the negotiation template", proposes an approach for evaluating negotiation offers when the negotiator's preferences are expressed verbally. This approach, called MARS (Measuring Attractiveness near Reference Situations), combines two multiple criteria decision making methods: ZAPROS and MACBETH.

The second paper "Integrating Time-preferences into E-Negotiation Systems: A Model, Elicitation Approach and Experimental Implications" authored by Venkataraghavan Krishnaswamy, Aseem Pahuja and R.P.Sundarraj, covers aspects of time preferences for electronic negotiations in the context of online marketplaces. This work shows that the time preference varies across buyers. The paper also discusses how different types of preferences can be elicited, represented and integrated with electronic negotiations.

In the third contribution, "Analyzing the multiple dimensions of negotiation processes", Michael Filzmoser, Patrick Hippmann and Rudolf Vetschera analyse three 
dimensions of negotiation processes: substantive, communicational and emotional. An approach is proposed that allows for the consideration of all these dimensions. An empirical study of the relations between these dimensions shows a strong linkage between communication behaviour and emotions and a weaker connection with the substantive dimension.

The fourth paper is dedicated to the effects of personalised negotiation training on learning and performance in electronic negotiations. Philipp Melzer and Mareike Schoop, in their paper "The Effects of Personalised Negotiation Training on Learning and Performance in Electronic Negotiations", propose an application of personalised end user training to negotiation support systems. The authors propose two personalised end-user- training approaches: enactive and vicarious training, in order to match learners' learning styles. This work contributes to the integration of theories of individual differences in electronic negotiations.

The fifth paper addresses problems of sensitive multi-unit assignment, where a set of resources has to be allocated amongst a set of agents having multi-unit demands. The paper "Using Bids, Arguments and Preferences in Sensitive Multi-Unit Assignments: A p-equitable Process and a Course Allocation Case Study", by Mireille Ducasse ?and Peggy Cellier, proposes an interactive process for multi-assignment problems where agents can give arguments for justifying their choices. The presented group decision support system is based on Logical Information Systems; enabling decision makers to handle bids, arguments and preferences in a unified interface.

Finally, Jorge E. Hernández, Andrew C. Lyons and Konstantinos Stamatopoulos, in their paper "A DSS-based framework for enhancing collaborative web-based operations management in manufacturing SME supply chains", propose a standard on-line based collaborative framework to support collaborative decision making in SMEs (small and medium-sized enterprises). This framework is applied to one precision engineering supply chain which is focused on supporting the manufacturing planning process through information sharing across the manufacturing SMEs, their customers and suppliers. The paper shows that through this framework, companies can enhance their decision-making process collaboratively and improve their management systems and operational practices.

\section{Conclusions}

In this special issue, a number of existing gaps, in terms of ways of considering dyadic and networked negotiations, as well as collaborative technologies, in organisations, have been addressed. Most of the approaches presented here feature holistic, personalised and multi-dimensional negotiations models. In addition, the collaborative decision-making approach is also discussed through preference elicitation and electronic negotiations based models. These approaches imply real implications for organisations and domains. In this context, contributions based on case studies include: entertainment retailers, movie tickets providers, European universities, courses allocation and precision engineering. The challenges faced by networked SME's are discussed and remedies proposed. 
Most of the papers included in this special issue have acknowledged the fact that, when more variables and constraints are linked to the decision-making process, the negotiation and collaboration may turn to be ineffective or inaccurate. Therefore, a suggestion is made to set the right application domain for the proposed collaborative decision-making solutions, which will imply organisations' tailored negotiation methods and collaborative tools.

To sum up, the contributions to this special issue serve as a springboard for the future research directions including the following areas: (1) establishing joint research activities to join negotiation and multi-criteria decision-making problems within the support of standard software tools, (2) applying multivariate techniques to analyse the relationships and interactions amongst the dimensions of the negotiation process simultaneously, (3) investigating the effect of negotiation processes on behavioural issues such as risk, magnitudes and preferences, (4) analysing negotiation tasks on more granular level to observe actual task-related behaviour of participants instead of measuring their potentially biased perceptions, (5) extending the use of collaborative tools to include complex adaptive systems in public sector and (6) applying negotiation methods, solutions and collaborative technologies in a variety of sectors.

Acknowledgments For this special issue edition, Guest Editors would like to thank the Group Decision \& Negotiation Journal Editor in-Chief, Prof. Gregory Kersten, for his valuable contributions and support across this guest editing process. In addition, a special thanks to Ms. Cynthia Clement, Springer Journal Editorial Office Assistant, for her support within the submission systems. Finally, Guest Editors would like to express their entire gratitude to all the reviewers who contributed to this special issue, without their contributions and professional works, which made this special issue possible. Hence, our especial thanks to: Adla, Abdelkader, University of Oran, Algeria Amblard, Frédéric, University of Toulouse, France Cellary, Wojciech, Poznan University of Economics, Poland Chan, Hing Kai, University of Nottingham, China Costa, Joao Paulo, University of Coimbra, Portugal Daher, Suzana, Federal University of Pernambuco, Brazil Dargam, Fatima, SimTech Simulation Technology, Austria Delibasic, Boris, University of Belgrade, Serbia and Montenegro Dias, Luis C, Universidade de Coimbra, Portugal Ducasse, Mireille, IRISA-INSA, France Eom, Sean, Southeast Missouri State University, United States Etezadi, Jamshid, Concordia University, Canada Gupta, Saurabh, University of North Florida, United States Kaminski, Bogumil, Warsaw School of Economics, Poland Kersten, Gregory E., Concordia University, Canada Kolfschoten, Gwendolyn L., Delft University of Technology, Netherlands Linden, Isabelle, University of Namur, Belgium Liu, Shaofeng, University of Plymouth, United Kingdom Morais, Danielle Costa, Federal University of Pernambuco, Brazil Munier, Bertrand, GRID, Management Science Dept, France Ortiz, Angel, Universitat Politècnica de València, Spain Papamichail, Nadia, Manchester Business School, United Kingdom Philips-Wren, Gloria, Loyola University Maryland, United States Stewart, Theodor J, University of Cape Town, South Africa Sundarraj, Rangaraja P., Indian Institute of Technology Madras, India Turel, Ofir, California State University, United States Zamfirescu, Constantin, University of Sibiu, Romania Zheng, Ronghuo, Carnegie Mellon University, United States

\section{References}

Azadegan A, Kolfschoten G (2014) An assessment framework for practicing facilitator. Group Decis Negot 23(5):1013-1045

Boddy D, Macbeth D, Wagner B (2000) Implementing collaboration between organizations: an empirical study of supply chain partnering. J Manag Stud 37(7):1003-1018

Cheung R, Vogel D (2013) Predicting user acceptance of collaborative technologies: an extension of the technology acceptance model for e-learning. Comput Educ 63:160-175

Daniels M, Cajander Å, Clear T, McDermott R (2015) Collaborative technologies in global engineering: new competencies and challenges. Int J Eng Educ 31(1):267-281 
Dean DL, Lee JD, Pendergast MO, Hickey AM, Nunamaker JF Jr (1997) Enabling the effective involvement of multiple users: methods and tools for collaborative software engineering. J Manag Inf Syst 14(3):179-222

Dean D, Orwig R, Vogel D (2000) Facilitation methods for collaborative modeling tools. Group Decis Negot 9(2): 109-128

De Vreede GJ, Boonstra J, Niederman F (2002) What is effective GSS facilitation? A qualitative inquiry into participants' perceptions. In 35th annual Hawaii international conference on system sciences (HICSS '02), vol 1: 43b

Kwok RCW, Ma J, Vogel D (2000) Effect of GSS and facilitation on knowledge restructuring. In: Proceedings of the 33rd Hawaii international conference on system sciences (IEEE), vol 1, pp 4-7

Lagroue HJ III (2008) The effectiveness of virtual facilitation for supporting group decision-making. Int J Inf Decis Sci 1(2):164-177

Tavella E, Franco LA (2015) Dynamics of group knowledge production in facilitated modelling workshops: an exploratory study. Group Decis Negot 24(3):451-475

Zaraté P, Kersten, GE, Hernández, JE (Eds.) (2014) Group decision and negotiation. A process-oriented view: joint INFORMS-GDN and EWG-DSS international conference, GDN 2014, Toulouse, France, 10-13 June 2014, Proceedings, LNBIP, vol 180, Springer 